\title{
Bounded Environment Passivity of the Classical Position-Force Teleoperation Controller.
}

\author{
B. Willaert, B. Corteville, D. Reynaerts, H. Van Brussel and E.B. Vander Poorten
}

\begin{abstract}
This paper derives analytic guidelines to tune the popular Position-Force bilateral controller and improve its performance by incorporating available knowledge on the bounds of the environment impedance. The proposed guidelines can prove especially useful in the domain of telesurgery where a need exists for well-understood bilateral teleoperation controllers, that show good performance and where many tasks can be characterized by restricted and relatively easily definable impedance regions. This paper firstly analyses the two-port passivity and absolute stability properties of two alternatives of the Position-Force controller. The limitations on achievable performance when guaranteeing absolute stability with arbitrary environments are detailed. Next, a novel method, called Bounded Environment Passivity method is introduced. This method enables the design of teleoperation controllers that show passive behaviour for interactions with an environment that varies over a given range of impedances. A set of guidelines that allow a smarter trade-off between performance and stability follows. The theoretical results are verified experimentally on a 1-d.o.f. teleoperation setup.
\end{abstract}

\section{INTRODUCTION}

This paper rigorously analyzes the stability properties of the well-known Position-Force bilateral controller [1], [2]. This controller appears under many different names in literature ${ }^{1}$. The Position-Force controller commands the slave robot to follow the position of the master robot and reflects the interaction force, measured at the slave side, to the master side. The success of the Position-Force controller lies in its simplicity. It is relatively easy to implement and shows a reasonably good performance. Its main limitation is its stability. Especially for stiff environments stability problems are being reported [1], [10], [11], [12].

Researchers have spent a considerable amount of effort to study the stability properties of the Position-Force controller. McAree et al. analyze the pole-locations of the closed loop system encompassing operator and environment [12], whereas Fite et al. apply loop-shaping techniques in [2]. Both works, however, rely on a fixed operator and a known environment. It is widely known that the behaviour of an operator is highly variable and difficult to predict, depending on factors such as posture, grip and fatigue [13], [11]. Also, in realistic teleoperation scenario's precise knowledge of the environment characteristics is missing. To cope with the

All authors are with the Department of Mechanical Engineering, K.U.Leuven, Celestijnenlaan 300C, B-3001 Heverlee, Belgium

Corresponding author: bert.willaert@mech.kuleuven.be

${ }^{1}$ Names in literature: Force-Position controller [3], [4], Position-Force architecture [1], [2], Force Reflection Type Controller [5], Forward Flow architecture [6], Kinesthetic Force Feedback controller [7], Direct Force Feedback controller [8] or Direct Force Reflection controller [9]. unpredictable behaviour of the operator and the unknown environment, Passivity Theory could be applied [14]. However, a well-known fact, which is confirmed analytically further in this paper, is that the Position-Force controller is non-passive. Another, less conservative but still sufficient condition for stable interaction with arbitrary passive environment and operator is the absolute stability property [15]. This property can be checked by calculating Llewellyn's absolute stability criteria [16], [17]. Tavakoli et al. proved analytically that a specific implementation of the Position-Force controller cannot be absolutely stable [9]. Hashtrudi-Zaad et al. investigated among other controllers the Position-Force controller based on a numerical evaluation of Llewellyn's absolute stability criteria [4]. However, none of the aforementioned works described analytic closed-form stability conditions, as presented further in this paper.

The Position-Force controller under study in this paper is popular when targeting soft environment manipulation, such as in telesurgery [18]. This controller is being applied for telesurgery in, among others, [7], [8] and [9]. Other works, such as [19], [20] and and [21] compare alternative controllers to the Position-Force controller. The popularity of the Position-Force controller in telesurgery can be explained by the need for a) well-understood b) bilateral teleoperation controllers, that c) show good performance. Although time delay is often mentioned as an important aspect of telesurgery [22], [23], this work does not deal with time delay. The authors are convinced that even without time delay, the design of performant teleoperation controllers remains a real challenge. Moreover, time delay is mostly not an issue in current telesurgical systems used in clinical practice, as the master and slave device are typically in the same room.

This paper presents a new method, which is called Bounded Environment Passivity. The proposed method runs parallel to efforts by [24], [25] and [26] which also incorporate bounds on the dynamic range of the environment and/or operator to obtain less conservative controllers. As stated in [14], pure springs and pure masses can be considered as the worst case environments for stability. In this work, the environment is considered to be a pure stiffness as in teleoperation, especially in telesurgery [8], displaying stiffnesses seems to be more relevant. Moreover, in telesurgery, one can easily delineate a maximum value of the environment stiffness. This knowledge is exploited here to come up with guidelines to tune the Position-Force controllers, allowing a 
more conscious trade-off between performance and stability.

Two alternatives of the Position-Force controller are described in section II. The Two-port Passivity and the Absolute Stability of these alternatives are studied in section III and section IV respectively. The novel method is described in section $\mathrm{V}$. The paper ends with experimental results in section VI, after which conclusions are drawn in section VII.

\section{The Position-Force CONTROLleR}

The analysis in this paper is based on 1-d.o.f rigid-body models for master and slave, obeying the following equations of motion:

$$
\begin{gathered}
F_{h}+\tau_{m}=M_{m} \ddot{x}_{m}+B_{m} \dot{x}_{m}, \\
\tau_{s}-F_{e}=M_{s} \ddot{x}_{s}+B_{s} \dot{x}_{s}, \\
Z_{m}=M_{m} s+B_{m}, \quad Z_{s}=M_{s} s+B_{s},
\end{gathered}
$$

with $Z_{m}$ and $Z_{s}$ representing the impedances of the master and the slave robot. Remark that for a rigid body model the positions $x_{m}$ and $x_{s}$ (the position of the motors) are equal to respectively $x_{h}$ and $x_{e}$ (the position of the end-effectors). The basic configuration of the Position-Force controller is illustrated in Fig. 1. The control inputs for the motors of the master and the slave are:

$$
\begin{gathered}
\tau_{m}=-\lambda \cdot F_{e} \\
\tau_{s}=C_{s}(s) \cdot\left(\mu \cdot x_{m}-x_{s}\right),
\end{gathered}
$$

with $\mu$ and $\lambda$ the position and force scaling factor. Note that here an impedance-type ${ }^{2}$ master is supposed and pure open-loop force control is used. The compensator $C_{s}(s)$ of the closed-loop position controller is typically a PDcompensator: $K_{v} s+K_{p}$. This configuration has been studied in [1], [4], [27], [9]. An alternative controller that appears in literature [5], [12], [28] differs in the fact that it does not contain a master velocity feedforward term. The control input for the slave is then given by:

$$
\tau_{s}=K_{p}\left(\mu x_{m}-x_{s}\right)-K_{v} \dot{x}_{s}
$$

As far as the authors are aware of, a concise comparison of the difference between both configurations has not yet been described. In this paper both configurations will be covered. For clarity the approach based on (5) will be referred to as the PD-F scheme, whereas the one based on (6) will be called the P-F scheme.

All numerical calculations and experiments reported in this paper are based and done on the 1-d.o.f. experimental masterslave setup shown in Fig. 9. The experimental setup consists

\footnotetext{
${ }^{2}$ An impedance-type device is characterized by low inertia and low friction/damping. A good example is the PHANToM haptic device.
}

\begin{tabular}{|c|c|c|c|}
\hline \multicolumn{2}{|c|}{ Model } & \multicolumn{2}{|c|}{ Controller } \\
\hline$M_{m}$ & $0.64 \mathrm{~kg}$ & $K_{v}:$ & $80 \mathrm{Ns} / \mathrm{m}$ \\
\hline$B_{m}:$ & $3.4 \mathrm{Ns} / \mathrm{m}$ & $K_{p}:$ & $4000 \mathrm{~N} / \mathrm{m}$ \\
\hline$M_{s}:$ & $0.61 \mathrm{~kg}$ & $\mu:$ & 1 \\
\hline$B_{s}:$ & $11 \mathrm{Ns} / \mathrm{m}$ & $\lambda:$ & 1 \\
\hline
\end{tabular}

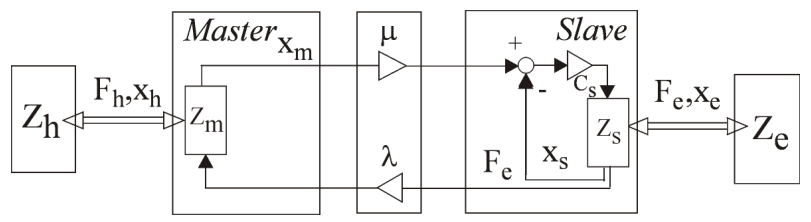

Fig. 1. Representation of the classic Position-Force controller (PD-F scheme)
TABLE I

PARAMETERS OF THE TELEOPERATION SYSTEM

of two current-driven voice coil motors from hard disk drives. On both devices, one-dimensional force sensors are mounted, measuring the interaction forces between slave and environment at one side, and between the human operator and the master at the other side (resolution: $0.05 \mathrm{~N}$ ). Linear encoders offer accurate position measurements (resolution: $1 \mu \mathrm{m})$. A rigid-body model for the master and the slave of this setup is justified because structural resonance frequencies are above $100 \mathrm{~Hz}$. Table I gives an overview of the used parameters for the model and the controller. These nominal parameters are based on the linear model identification of the real setup and on the implementation of the controllers on this setup. The controllers are implemented on a dSpace board, in a real time loop with a frequency of $1 \mathrm{kHz}$.

\section{TWO-PORT PASSIVITY ANALYSIS}

Passivity has turned out to be an interesting property for the study of human-robot interaction [29], [30]. In the case of teleoperation, the robotic system contains both a master and slave robot, which interact with the human operator at the master side and the external environment at the slave side. Fig. 2 shows a network model of such a teleoperation system. To evaluate the passivity of the teleoperation system, one can look at its scattering matrix [14], or make use of the Raisbeck passivity criteria [31] based on the elements $h_{i j}$ of the hybrid matrix [6] of the teleoperation system:

- no $h_{i j}$ has poles in the right half plane,

- any poles of the $h$-parameters $h_{11}, h_{12}, h_{21}$ and $h_{22}$ on the imaginary axis are simple, and the residues $d_{11}, d_{12}$, $d_{21}$ and $d_{22}$ at these poles satisfy the conditions: $d_{11} \geq 0$, $d_{22} \geq 0$ and $d_{11} d_{22}-d_{12} d_{21} \geq 0$ with $d_{21}=d_{12}^{*}$, and

- the real $\left(r_{i j}=\mathfrak{R}\left(h_{i j}\right)\right)$ and imaginary $\left(i_{i j}=\mathfrak{I}\left(h_{i j}\right)\right)$ parts of the $h_{i j}$-elements satisfy:

$$
\begin{gathered}
r_{11} \geq 0, \quad r_{22} \geq 0, \forall \omega \\
4 r_{11} r_{22}-\left(r_{12}+r_{21}\right)^{2}-\left(i_{12}-i_{21}\right)^{2} \geq 0, \forall \omega .
\end{gathered}
$$

In the case of the Position-Force controller the elements of the hybrid matrix are found to be:

$$
\begin{aligned}
& h_{11}=M_{m} s+B_{m}, \quad h_{12}=\lambda, \\
& h_{21}=\frac{-\mu K_{p}}{M_{s} s^{2}+\left(B_{s}+K_{v}\right) s+K_{p}}, \quad h_{22}=\frac{s}{M_{s} s^{2}+\left(B_{s}+K_{v}\right) s+K_{p}} \text {. }
\end{aligned}
$$

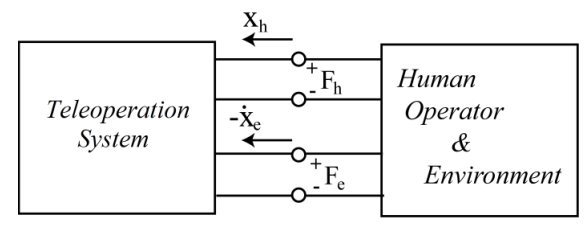

Fig. 2. A two-port network representation of a teleoperation system 
for the P-F scheme, whereas the PD-F scheme leads to the following hybrid parameters:

$$
\begin{array}{llrl}
h_{11} & =M_{m} s+B_{m}, & & h_{12}=\lambda, \\
h_{21} & =\frac{-\mu\left(K_{v} s+K_{p}\right)}{M_{s} s^{2}+\left(B_{s}+K_{v}\right) s+K_{p}}, & & h_{22}=\frac{s}{M_{s} s^{2}+\left(B_{s}+K_{v}\right) s+K_{p}},
\end{array}
$$

For both hybrid matrices, it is relatively straightforward to see that all conditions except (8) hold. After performing some algebraic manipulations, (8) can be written as:

$$
-\lambda^{2} M_{s}^{2} \omega^{4}+a_{2} \omega^{2}-K_{p}(\lambda-\mu)^{2} \geq 0, \forall \omega
$$

with

$$
\begin{aligned}
a_{2}= & 4 B_{m}\left(B_{s}+K_{v}\right)-\lambda^{2}\left(B_{s}+K_{v}\right)^{2}-\mu^{2} K_{v}^{2} \\
& +2 \mu \lambda K_{p} M_{s}(\lambda-\mu)+2 \mu \lambda K_{v}\left(B_{s}+K_{v}\right)
\end{aligned}
$$

for the PD-F scheme. One can easily verify that this inequality in $\omega$ can only hold in the trivial case that $M_{s}=B_{s}=0^{3}$. Note that a similar derivation can be made for the P-F scheme. The above derivation allows the formulation of the following theorem:

THEOREM 1 (Active Position-Force Controller): The Position-Force controllers defined by (4) and (5) or by (4) and (6) form a non-passive system when applied upon the teleoperator (1) and (2) for non-trivial parameters of the system.

Actually, passivity is a conservative property as it neglects the fact that no direct interaction between operator and environment occurs. The next section analyzes the absolute stability property which is a less conservative property incorporating this structural knowledge.

\section{Absolute Stability Analysis}

A linear two-port is absolutely or unconditionally stable if and only if there is no set of passive one-ports for which the coupled system is unstable. Llewellyn's stability criteria provide necessary and sufficient conditions for absolute stability [16]. These criteria are equivalent to the following conditions in terms of the immittance matrix $P$ :

- the immittance parameters $p_{11}$ and $p_{22}$ have no poles in the open right-half-plane (RHP),

- any poles of $p_{11}$ and $p_{22}$ on the imaginary axis are simple and have real and positive residues, and

$$
\begin{gathered}
\Re\left(p_{11}\right) \geq 0, \forall \omega \\
\eta(\omega)=-\cos \left(\angle p_{12} p_{21}\right)+2 \frac{\mathfrak{R}\left(p_{11}\right) \Re\left(p_{22}\right)}{\left|p_{12} p_{21}\right|} \geq 1, \forall \omega .
\end{gathered}
$$

These conditions are valid for any member of the immittance class. Here, the conditions are checked for the hybrid matrices (10) and (9). For both hybrid matrices, it is relatively straightforward to see that all conditions stated above, except (14), hold. The following subsections elaborate further on this inequality (14) for both the P-F and the PD-F scheme.

\footnotetext{
${ }^{3}$ Note that (11) can be written as $a x^{2}+b x+c \geq 0, \forall x \geq 0$ where $x=\omega^{2}$ is used. This holds $\Leftrightarrow a \geq 0$ and $c \geq 0$ and $\left(b \geq 0\right.$ or $\left.b^{2}-4 a c \leq 0\right)$.
}

\section{A. Absolute stability of the P-F scheme}

The parameter $\eta(\omega)$ in inequality (14) can be written as the sum of $\eta_{1}(\omega)$ and $\eta_{2}(\omega)$. For the P-F scheme these two parameters can be written as:

$$
\begin{array}{r}
\eta_{1}(\omega)=\cos \left(\angle \frac{\mu \lambda K_{p}}{M_{s} s^{2}+\left(B_{s}+K_{v}\right) s+K_{p}}\right), \\
\eta_{2}(\omega)=2 \frac{\mathfrak{R}\left(M_{m} s+B_{m}\right) \mathfrak{R}\left(\frac{s}{M_{s} s^{2}+\left(B_{s}+K_{v}\right) s+K_{p}}\right)}{\left|\frac{-\mu \lambda K_{p}}{M_{s} s^{2}+\left(B_{s}+K_{v}\right) s+K_{p}}\right|} .
\end{array}
$$

Replacing $s$ by $j \omega$ results in the following analytic equations:

$$
\begin{gathered}
\eta_{1}(\omega)=\frac{K_{p}-M_{s} \omega^{2}}{\sqrt{\left(K_{p}-M_{s} \omega^{2}\right)^{2}+\left(B_{s}+K_{v}\right)^{2} \omega^{2}}}, \\
\eta_{2}(\omega)=\frac{2 B_{m}\left(B_{s}+K_{v}\right) \omega^{2}}{\mu \lambda K_{p} \sqrt{\left(K_{p}-M_{s} \omega^{2}\right)^{2}+\left(B_{s}+K_{v}\right)^{2} \omega^{2}}} .
\end{gathered}
$$

Based on these analytic equations, it turns out that the condition (14) only holds when the parameters of the system satisfy the following conditions:

$$
\begin{gathered}
\mu \lambda \leq 4 \frac{B_{m}}{\left(B_{s}+K_{v}\right)}, \\
\mu \lambda \leq \frac{B_{m}\left(B_{s}+K_{v}\right)}{M_{s} K_{p}} .
\end{gathered}
$$

Fig. 3 illustrates these conditions for the parameters of Table I. All sets of parameters belonging to the shaded area result in an absolutely stable system. The absolute maximum value of $\mu \lambda$ is reached for a critically damped slave robot $\left(B_{s}+K_{v}=2 \sqrt{M_{s} K_{p}}\right)$. For the parameters of Table I, the maximum value of $\mu \lambda$ is e.g. 0.127 , which clearly restricts the transparency of the system. The above results are summarized in the following theorem:

\section{THEOREM 2 (Absolutely stable P-F controllers):}

The Position-Force controller without master velocityfeedforward, applied upon the teleoperator (1), (2) and (3), and expressed by control laws (4) and (6) is absolutely stable when the scale factors $\lambda$ and $\mu$ respect both inequalities (19) and (20).

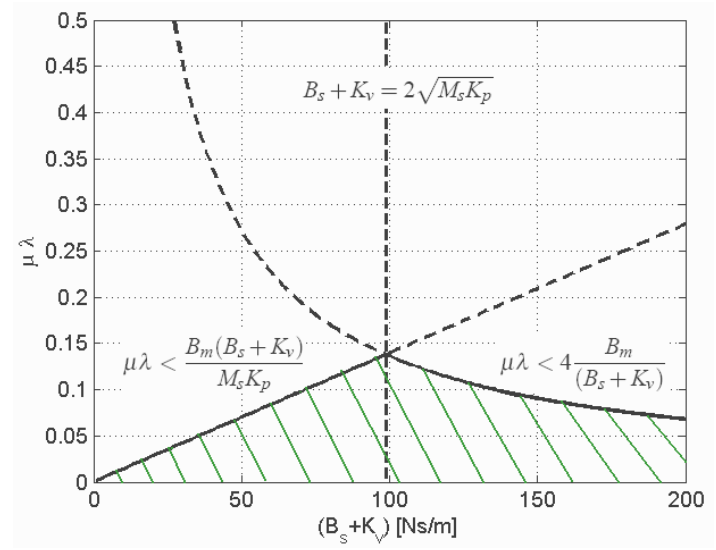

Fig. 3. Conditions on the parameters of the system resulting in an absolute stable system for the P-F scheme. 

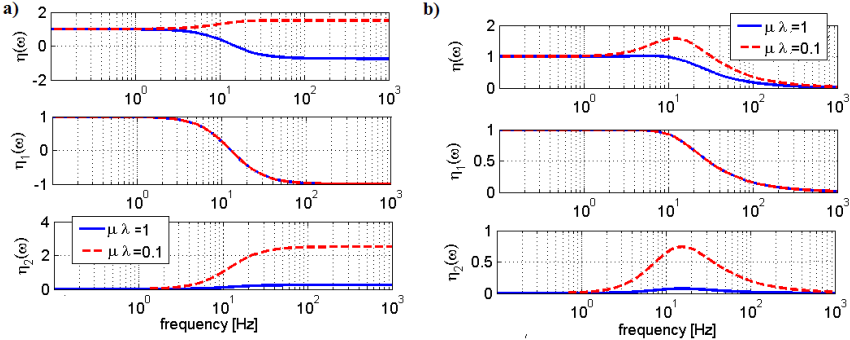

Fig. 4. The parameters $\eta(\omega), \eta_{1}(\omega)$ and $\eta_{2}(\omega)$ for two different values of $\mu \lambda$ for (a) the P-F scheme and (b) the PD-F scheme.

Fig. 4(a) shows the parameters $\eta(\omega), \eta_{1}(\omega)$ and $\eta_{2}(\omega)$ for two different values of $\mu \lambda$ and the parameters of Table I. For $\mu \lambda=0.1$ it can be checked that the two conditions are satisfied, thus the system with $\mu=0.1$ is absolutely stable. This can be seen in Fig. 4(a) as $\eta(\omega) \geq 1$ holds for all frequencies.

\section{B. Absolute stability of the PD-F scheme}

For the PD-F scheme, the parameter $\eta(\omega)$ can again be written as the sum of $\eta_{1}(\omega)$ and $\eta_{2}(\omega)$, with:

$$
\begin{aligned}
& \eta_{1}(\omega)=\cos \left(\angle \frac{\mu \lambda\left(K_{v} s+K_{p}\right)}{M_{s} s^{2}+\left(B_{s}+K_{v}\right) s+K_{p}}\right), \\
& \eta_{2}(\omega)=2 \frac{\Re\left(M_{m} s+B_{m}\right) \mathfrak{R}\left(\frac{s}{M_{s} s^{2}+\left(B_{s}+K_{v}\right) s+K_{p}}\right)}{\left|\frac{-\mu \lambda\left(K_{v} s+K_{p}\right)}{M_{s} s^{2}+\left(B_{s}+K_{v}\right) s+K_{p}}\right|} .
\end{aligned}
$$

Replacing $s$ by $j \omega$ results in the following analytic equations:

$$
\begin{gathered}
\eta_{1}(\omega)=\operatorname{sign}(\mu \lambda) \frac{K_{p}^{2}+\left(K_{V}^{2}+K_{v} B_{S}-M_{s} K_{p}\right) \omega^{2}}{\sqrt{\left(K_{p}-M_{S} \omega^{2}\right)^{2}+\left(\left(B_{S}+K_{v}\right) \omega\right)^{2}} \sqrt{K_{p}^{2}+K_{v}^{2} \omega^{2}}}, \\
\eta_{2}(\omega)=\frac{2 B_{m}\left(B_{S}+K_{v}\right) \omega^{2}}{|\mu \lambda| \sqrt{\left(K_{p}-M_{S} \omega^{2}\right)^{2}+\left(\left(B_{S}+K_{v}\right) \omega\right)^{2}} \sqrt{K_{p}^{2}+K_{v}^{2} \omega^{2}}} .
\end{gathered}
$$

From these equations follows that for $\omega \rightarrow \infty, \eta_{1}(\omega) \rightarrow 0$ and $\eta_{2}(\omega) \rightarrow 0$, independent of the parameters of the system. This means that (14) does not hold for all $\omega$, leading to the following result:

\section{THEOREM 3 (Non absolutely stable PD-F controller):}

The Position-Force controller with master velocityfeedforward, applied upon the teleoperator (1), (2) and (3), and expressed by control laws (4) and by (5) is never absolutely stable when $M_{s} \neq 0$.

Fig. 4(b) shows $\eta(\omega), \eta_{1}(\omega)$ and $\eta_{2}(\omega)$ for two different values of $\mu \lambda$ and the parameters of Table I.

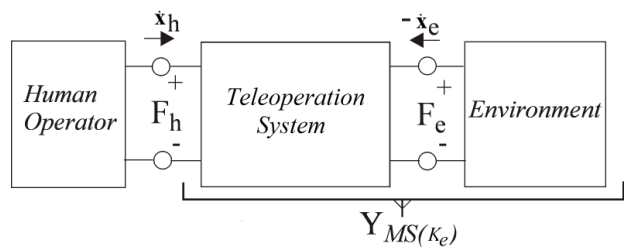

Fig. 5. A one-port network $Y_{M S\left(K_{e}\right)}$ representation of a combined teleoperator-environment system.

\section{ONE-PORT PASSIVITY ANALYSIS}

The above derivation showed that stable interaction can be achieved with any passive environment for the PositionForce controller, but only for very limited values of $\mu \lambda$ in the case of $\mathbf{P}-\mathbf{F}$ and even never in the case of PD-F. In this section, a novel method is proposed that allows to improve the tuning of the Position-Force controller, by making use of available knowledge about the environment. The new method is also based on the passivity concept, but in contrast to section III, here, the dynamics of the master, slave, controller and environment are combined into one one-port network $Y_{M S\left(K_{e}\right)}$, as shown in Fig. 5. Next, the coupled stability between any operator and the $Y_{M S\left(K_{e}\right)}$ one-port is discussed. Coupled stability can be checked by verifying positive realness of the admittance $Y_{M S\left(K_{e}\right)}$ [14]. In order to determine $Y_{M S\left(K_{e}\right)}$, an assumption has to be made about the environment. As stated in[14], pure springs and pure masses can be considered as the worst case environments since their admittance is not strictly passive. Since in teleoperation, especially in telesurgery, displaying stiffnesses is in most cases more relevant, in this work the environment is considered to be a pure spring with stiffness $K_{e}$. Through this assumption the admittance of the one-port for the P-F scheme can be written as:

$$
Y_{M S\left(K_{e}\right)}=\frac{s\left(M_{s} s^{2}+\left(B_{s}+K_{v}\right) s+\left(K_{p}+K_{e}\right)\right)}{\left(M_{m} s^{2}+B_{m} s\right)\left(M_{s} s^{2}+\left(B_{s}+K_{v}\right) s+\left(K_{p}+K_{e}\right)\right)+\mu \lambda K_{e} K_{p}} .
$$

For the PD-F scheme this yields:

$$
Y_{M S\left(K_{e}\right)}=\frac{s\left(M_{s} s^{2}+\left(B_{s}+K_{v}\right) s+\left(K_{p}+K_{e}\right)\right)}{\left(M_{m} s^{2}+B_{m} s\right)\left(M_{s} s^{2}+\left(B_{s}+K_{v}\right) s+\left(K_{p}+K_{e}\right)\right)+\mu \lambda K_{e}\left(K_{v} s+K_{p}\right)} .
$$

The proposed approach, referred to as Bounded Environment Passivity, results in conditions to calculate controllers that make the combined system $Y_{M S\left(K_{e}\right)}$ behave 'passively' for a range of $\left[K_{e}=0-K_{e}^{\max }\right]$. The derived expressions provides limits to the achievable scale factors $\mu \lambda$ as a function of the range on $K_{e}$ and as a function of system and control parameters. First derivations are done for the P-F, then for the PD-F scheme.

\section{A. Bounded Environment passivity for the $\boldsymbol{P}-\boldsymbol{F}$ scheme}

The $Y_{M S\left(K_{e}\right)}$ of the P-F scheme can be checked analytically. $\mathfrak{R}\left(Y_{M S\left(K_{e}\right)}(j \omega)\right) \geq 0$ can be expressed as:

$$
\begin{array}{r}
\left(M_{s}^{2} B_{m}\right) \omega^{6}+\left(B_{m}\left(B_{s}+K_{v}\right)^{2}-2\left(K_{p}+K_{e}\right) M_{s} B_{m}\right) \omega^{4}+ \\
\left(B_{m}\left(K_{p}+K_{e}\right)^{2}-\mu \lambda K_{e} K_{p}\left(B_{s}+K_{v}\right)\right) \omega^{2} \geq 0, \quad \forall \omega \geq 0,
\end{array}
$$

which results in the following conditions on the system parameters:

$$
\begin{aligned}
& B^{\text {lim }}=\min \left[\sqrt{2\left(K_{p}+K_{e}^{\max }\right) M_{s}}, 2 \sqrt{K_{p} M_{s}}\right] \\
& 0 \leq\left(B_{s}+K_{v}\right) \leq B^{l i m} \quad: \quad \mu \lambda \leq \frac{B_{m}\left(B_{S}+K_{v}\right)}{M_{S} K_{p}}\left[1+\frac{K_{p}}{K_{e}^{\max }}-\frac{\left(B_{S}+K_{v}\right)^{2}}{4 K_{e}^{\max } M_{S}}\right] \\
& \left(B_{s}+K_{v}\right) \geq B^{\text {lim }} \text { and } K_{e}^{\max } \leq K_{p} \quad: \quad \mu \lambda \leq \frac{B_{m}\left(K_{p}+K_{e}^{\max }\right)^{2}}{\left(B_{S}+K_{v}\right) K_{p} K_{e}^{\max }} \\
& \left(B_{s}+K_{v}\right) \geq B^{\lim } \text { and } K_{e}^{\max }>K_{p} \quad: \quad \mu \lambda \leq \frac{4 B_{m}}{\left(B_{S}+K_{v}\right)}
\end{aligned}
$$

Fig. 6 illustrates these conditions for the values in Table I and for different values of the selected maximum environment stiffness $K_{e}^{\max }(1000, \ldots, 16000 \mathrm{~N} / \mathrm{m})$. Note that solutions belonging to the area below the curves are valid. 


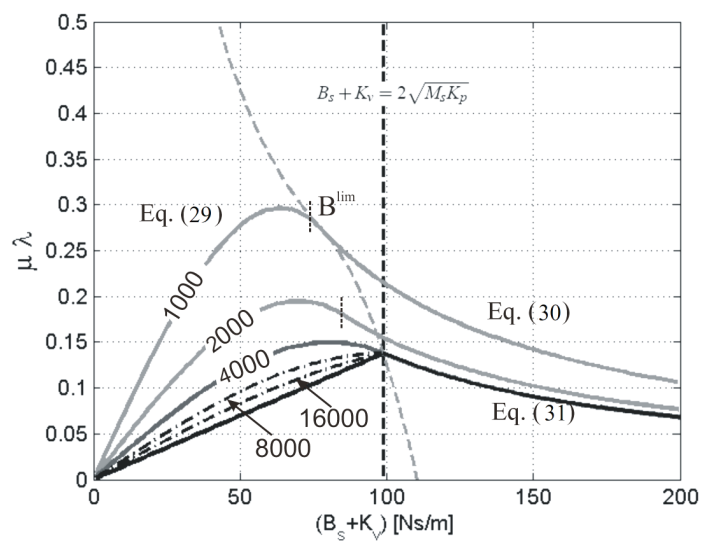

Fig. 6. The P-F scheme: conditions for bounded environment passivity for different values of $K_{e}^{\max }[1000-\infty \mathrm{N} / \mathrm{m}]$.

The maximum attainable value $\mu \lambda^{\max }$ for a given $K_{e}^{\max }$ is reached at different values of $B_{s}+K_{v}$. For $K_{e}^{\max }<2 K_{p}, \mu \lambda^{\text {max }}$ is found for:

$$
\left(B_{s}+K_{v}\right)^{\max }=\sqrt{\frac{4 M_{s}\left(K_{e}^{\max }+K_{p}\right)}{3}},
$$

with:

$$
\mu \lambda^{\max }=\frac{4}{3} \frac{B_{m}}{\sqrt{M_{s}} K_{p} K_{e}^{\max }} \sqrt{\frac{\left(K_{e}^{\max }+K_{p}\right)^{3}}{3}} .
$$

When $K_{e}^{\max } \geq 2 K_{p}$, maximal $\mu \lambda$ is reached for a critically damped slave and equals $2 B_{m} / \sqrt{M_{s} K_{p}}$ (this follows from (29), (32) and (33)). Note that this value corresponds to the value found for absolute stability in subsection IV-A. Thus, no gain in the factor $\mu \lambda$ can be achieved for the P-F scheme by using Bounded Environment Passivity when $K_{e}^{\max } \geq 2 K_{p}$. For smaller $K_{e}^{\max }$ e.g. $1000 N / m, \mu \lambda^{\max }$ is 0.29 , compared to the absolute maximum value of 0.137 reached under the condition of absolute stability.

Based on (33) the effect of the various parameters on $\mu \lambda^{\max }$ were calculated and plotted in Fig. 7(a). The figure shows the relation between the selected maximum environment stiffness $K_{e}^{\max }$ and $\mu \lambda^{\max }$ as well as the effect of $M_{s}, B_{m}$ and $K_{p}$ for a fixed maximum environment stiffness $K_{e}^{\max }=$ $1000 \mathrm{~N} / \mathrm{m}$. One can see that:

- $K_{e}^{\max }$ : a significant increase in allowable $\mu \lambda$ can only be obtained when the maximum environment stiffness $K_{e}^{\max }$ is limited. For $K_{e}^{\max } \rightarrow \infty$, the curve evolves towards the condition of absolute stability (derived in subsection IVA).
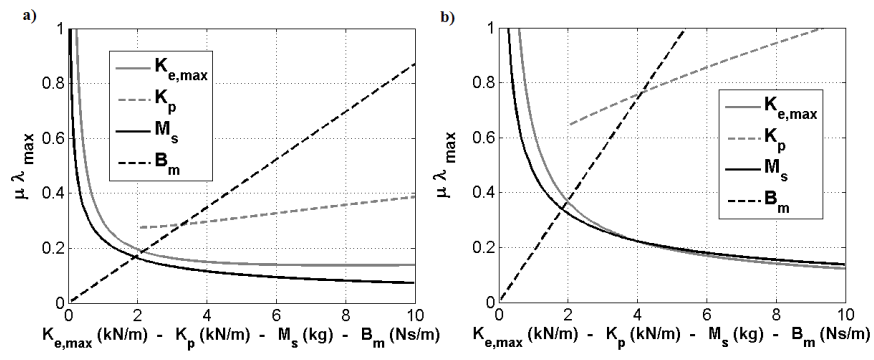

Fig. 7. Effect of $K_{e}^{\max }$ on $\mu \lambda^{\max }$ and the effect of $M_{s}, B_{m}$ and $K_{p}$ on $\mu \lambda^{\max }$ when $K_{e}^{\max }=1000$ for a) the P-F scheme and b) the PD-F scheme.
- $M_{s}$ : reductions of the slave mass are very effective to realize passivity with larger values of $\mu \lambda$.

- $B_{m}$ : an increase of master damping allows larger values of $\mu \lambda$. However, extra damping jeopardizes the system's transparency, causing a sluggish feeling.

- $K_{p}$ : also, a tighter position loop at the slave is found to increase maximal $\mu \lambda$.

\section{B. Bounded Environment passivity for the PD-F scheme}

The $\mathfrak{R}\left(Y_{M S\left(K_{e}\right)}(j \omega)\right) \geq 0$ condition for P-DF can be expressed as:

$$
\begin{aligned}
& \left.\left(M_{s}^{2} B_{m}\right) \omega^{6}+\left(B_{m}\left(B_{s}+K_{v}\right)^{2}-2\left(K_{p}+K_{e}\right) M_{s} B_{m}-\mu \lambda M_{s} K_{v} K_{e}\right)\right) \omega^{4} \\
& +\left(B_{m}\left(K_{p}+K_{e}\right)^{2}+\mu \lambda K_{v} K_{e}^{2}-\mu \lambda K_{e} K_{p} B_{s}\right) \omega^{2} \geq 0, \quad \forall \omega \geq 0 .
\end{aligned}
$$

Similar to subsection $\mathrm{V}-\mathrm{A}$, this condition can be reformulated to a set of conditions upon the parameters. Here, these conditions are rather complicated and they can be found in the Appendix. However, for $K_{v} \leq\left(M_{s} K_{p}\right) / B_{s}$, the conditions simplify to the following:

$\mu \lambda \leq \frac{B_{m}\left(B_{s}+K_{v}\right)}{M_{s} K_{e} K_{v}^{2}}\left(\left(B_{s}+K_{v}\right) K_{v}-2 M_{s} K_{p}+2 \sqrt{M_{s}^{2} K_{p}^{2}+M_{s} K_{v}^{2} K_{e}-K_{p} K_{v} B_{s} M_{s}}\right)$

Fig. 8 illustrates this relation for the parameters of Table I and $K_{e}^{\max }(1000, \ldots, 27000 N / m)$. In order to compare the PDF scheme with the P-F scheme, the curves delimiting the region of bounded environment passivity for the P-F scheme are shown again. For relatively low values of $K_{e}^{\max }$ the PDF scheme outperforms the P-F scheme. For a maximum environment stiffness $K_{e}^{\max }=1000 \mathrm{~N} / \mathrm{m}$ and $K_{v}=88 \mathrm{Ns} / \mathrm{m}$ e.g., $\mu \lambda=0.67$ (compared to 0.137 and 0.29 for respectively absolute stability and bounded environment passivity of the P-F scheme).

Based on (35), Fig. 7(b) shows the effect on the maximum allowed $\mu \lambda$ of the selected $K_{e}^{\max }$ as well as the effect of $M_{s}, B_{m}$ and $K_{p}$ for a fixed maximum environment stiffness $K_{e}^{\max }=1000 \mathrm{~N} / \mathrm{m}$. Note that the relation $K_{p}-\mu \lambda$ shown here, supposes a fixed damping ratio of the slave. Similar relations as in Fig. 7(a) appear for the dependency on $K_{e}^{\max }, M_{s}, B_{m}$ and $K_{p}$. The main difference with the $\mathbf{P}-\mathbf{F}$ scheme is that higher values of $\mu \lambda$ can be achieved for a certain value of $K_{e}^{\max }, M_{s}$ and $B_{m}$.

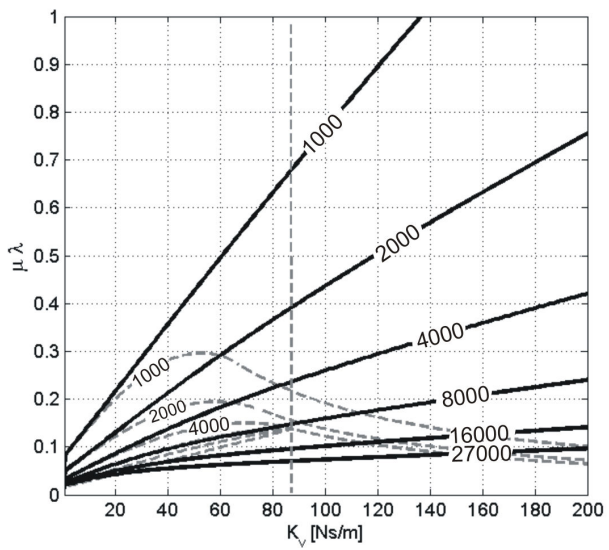

Fig. 8. Comparison between the conditions for bounded environment passivity for the P-F scheme (dashed) and the PD-F scheme (solid). 


\section{EXPERIMENTAL RESULTS}

Section $\mathrm{V}$ showed how to select a value for $\mu \lambda$ in order to guarantee stable interaction for any passive operator, based upon the selected value $K_{e}^{\max }$. The correctness of the proposed analytic conditions was numerically and experimentally verified. The experiments presented here study the inverse problem: the passivity of $Y_{M S\left(K_{e}\right)}$ is checked for different $K_{e}$ for $\mu=\lambda=1$, as $\mu \lambda=1$ is in favour of transparency.

As a human operator tends to stabilize the interaction, stable interaction with a human operator is not a sufficient demonstration of passivity $Y_{M S\left(K_{e}\right)}$. To check the passivity, the operator should behave as a worst case operator [14]. To approximate such behaviour, the system is checked for three different operators: a human operator holding the master, a soft spring fixed between a rigid wall and the master and a hard object contacting the master. For each of these 'operators', the stability is checked for four different environments: three springs fixed between a rigid wall and the slave $\left(K_{e}=100,300\right.$ and $\left.880 \mathrm{~N} / \mathrm{m}\right)$ and one hard contact $\left(K_{e}=27000 \mathrm{~N} / \mathrm{m}\right)$. The parameters for both control schemes are those in Table I, except for $K_{v}$ for the P-F scheme. This parameter is selected based on (32). For $K_{e}^{\max }=100 \mathrm{~N} / \mathrm{m}$ for example, this results in $K_{v}=58 \mathrm{Ns} / \mathrm{m}$. Fig. 10 shows the calculated $\Re\left(Y_{M S\left(K_{e}\right)}(j \omega)\right), \omega \in[1-100] H z$, for the different environments for both the PD-F and the P-F scheme.

Table II shows for each environment/controller combination, whether $Y_{M S\left(K_{e}\right)}$ is passive for $\mu=\lambda=1$, what $\mu \lambda^{\max }$ is and how the system behaves for the different operators. For the spring and hard contact operator, the system was considered stable, if a small, manually given, force input at the master did not result in growing oscillations. As can be expected, none of the 'operators' could cause an instability when the one-port is passive. However, for an active oneport the findings are less straightforward, as the stability depends on the operator. Remark the case with $K_{e}=300 \mathrm{~N} / \mathrm{m}$ for the P-F scheme. Although the system is clearly active, no human operator experiences an instability. This suggests that the current approach of guaranteeing stability for any

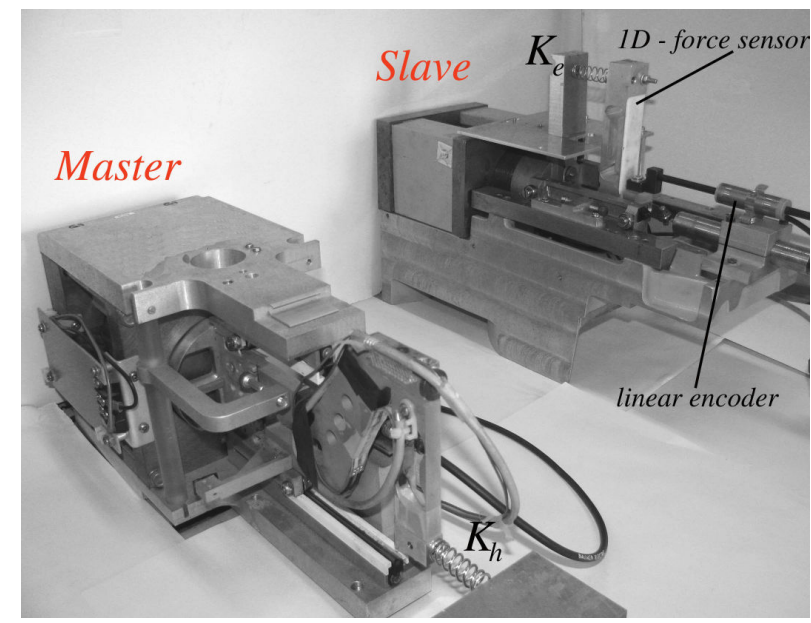

Fig. 9. The 1 d.o.f experimental teleoperation setup in the case that both the environment and the operator are a pure stiffness $\left(K_{e}\right.$ and $\left.K_{h}\right)$.
TABLE II

NUMERICAL AND EXPERIMENTAL RESULTS

\begin{tabular}{|c|c|c|c|c|}
\hline \multirow[t]{5}{*}{ P-F: } & $\overline{K_{e}}$ & Num & $\mu \lambda_{K_{y}=58}^{\max }$ & Exp \\
\hline & $100 \mathrm{~N} / \mathrm{m}$ & Passive & 2.2 & $\begin{array}{l}\text { Stable for } \\
\text { all operators }\end{array}$ \\
\hline & $300 \mathrm{~N} / \mathrm{m}$ & $\begin{array}{c}\text { Active } \\
\text { (peak at } 3 \mathrm{~Hz} \text { ) }\end{array}$ & 0.78 & $\begin{array}{l}\text { Unstable for } \\
\text { soft spring }\end{array}$ \\
\hline & $880 \mathrm{~N} / \mathrm{m}$ & $\begin{array}{c}\text { Active } \\
\text { (peak at } 5 \mathrm{~Hz} \text { ) }\end{array}$ & 0.32 & $\begin{array}{l}\text { Unstable for } \\
\text { soft spring }\end{array}$ \\
\hline & $27000 \mathrm{~N} / \mathrm{m}$ & $\begin{array}{c}\text { Active } \\
\text { (peak at } 12 \mathrm{~Hz} \text { ) }\end{array}$ & 0.13 & $\begin{array}{l}\text { Unstable for } \\
\text { all operators }\end{array}$ \\
\hline \multirow[t]{5}{*}{ PD-F: } & $\overline{\overline{K_{e}}}$ & $\overline{\text { Num }}$ & $\bar{\mu} \mu \lambda_{K_{y}=80}^{\max }$ & $\overline{\overline{E x p}}$ \\
\hline & $100 \mathrm{~N} / \mathrm{m}$ & Passive & 5.14 & $\begin{array}{l}\text { Stable for } \\
\text { all operators }\end{array}$ \\
\hline & $300 \mathrm{~N} / \mathrm{m}$ & Passive & 1.81 & $\begin{array}{c}\text { Stable for } \\
\text { all operators }\end{array}$ \\
\hline & $880 \mathrm{~N} / \mathrm{m}$ & $\begin{array}{c}\text { Active } \\
\text { (peak at } 11 \mathrm{~Hz} \text { ) }\end{array}$ & 0.7 & $\begin{array}{l}\text { Stable for } \\
\text { all operators }\end{array}$ \\
\hline & $27000 \mathrm{~N} / \mathrm{m}$ & $\begin{array}{c}\text { Active } \\
\text { (peak at } 34 \mathrm{~Hz} \text { ) }\end{array}$ & 0.07 & $\begin{array}{l}\text { Only stable for } \\
\text { soft spring }\end{array}$ \\
\hline
\end{tabular}
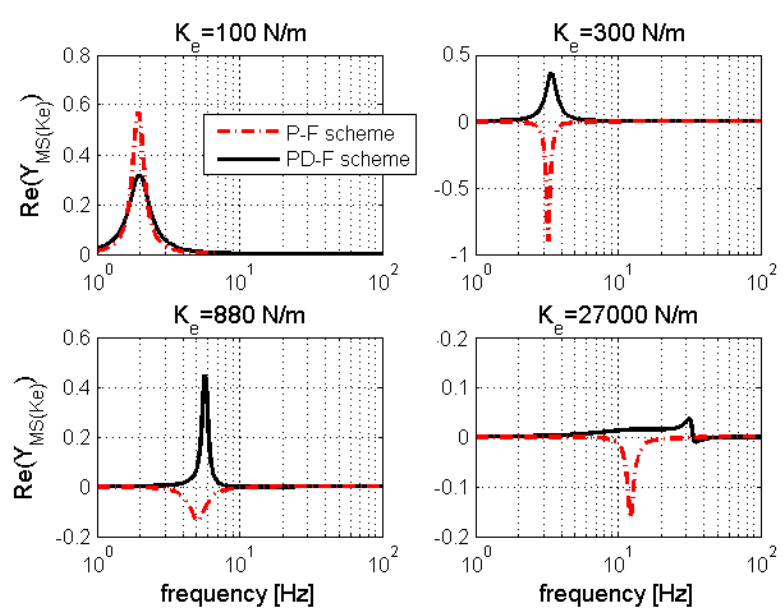

Fig. 10. Comparison between $\mathfrak{R}\left(Y_{M S\left(K_{e}\right)}(j \omega)\right)$ for the PD-F scheme and the P-F scheme for different values of $K_{e}$.

passive operator might still be too conservative. This will be investigated in further research. Lastly, these experiments confirm the finding in section V-B that the PD-F scheme has better stability properties.

\section{CONCLUSIONS}

In this paper, a detailed stability analysis of two alternatives of the classical Position-Force controller is presented. The analysis is based on two existing methods, i.e. Two-port Passivity and Absolute Stability and a new method which is referred to as Bounded Environment Passivity.

Firstly, it is proven analytically that the Position-Force controller is a non-passive controller. Secondly, it is analytically proven that for the PD-F scheme, absolute stability can never be guaranteed and that for the P-F scheme absolute stability can only be guaranteed for very limited scaling factors $\mu \lambda$. Next, the new bounded environment passivity approach is explained. This method makes use of the known/selected restrictions on the environment impedance. The environment is considered to be a pure stiffness. The passivity of the oneport $Y_{M S\left(K_{e}\right)}$ with which the operator interacts, is investigated for environment properties within the predescribed bounded region. This results in analytical guidelines to tune the Position-Force controller as a function of the selected range of environments. Two important findings are that: (1) the 
larger the maximum stiffness of the environment, the harder the restriction on $\mu \lambda$ and (2) reducing the inertia of the slave robot is an effective way to obtain larger maximal $\mu \lambda$-values. Moreover, the analysis pushes the PD-F scheme forward as the scheme with most possibilities to select a smart trade-off between performance and stability. Experiments confirm this finding.

In future work, the stability properties of extensions of the Position-Force controller, these can be 3- or 4-channel controllers, will be investigated following the same methodology. Also a method to deal with contacts that lie outside the hypothesized region of interest need to be further developed.

\section{ACKNOWLEDGMENTS}

This work was supported by a $\mathrm{PhD}$ grant from the Institute for the Promotion of Innovation through Science and Technology in Flanders (I.W.T.-Vlaanderen), two I.W.T projects (IWT/OZM/080086 and IWT/OZM/080003) and by the K.U.Leuven BOF-IDO/05/008 project as well as by an FP7-People Marie Curie Reintegration Grant, PIRG03-2008231045.

\section{APPENDIX}

This appendix shows the full analytic conditions resulting from the requirement that $\mathfrak{R}\left(Y_{M S\left(K_{e}\right)}\right) \geq 0$ for the PD-F scheme: [(36) or (37)] and [(38) or [(39) and (40)]]:

$$
\begin{gathered}
K_{e}^{\max } \leq \frac{B_{s} K_{p}}{B_{s}+2 K_{v}} \Rightarrow \mu \lambda \leq \frac{B_{m}\left(K_{e}^{\max }+K_{p}\right)^{2}}{\left(B_{s} K_{p}-K_{v} K_{e}^{\max }\right) K_{e}^{\max }} \\
K_{e}^{\max }>\frac{B_{s} K_{p}}{B_{s}+2 K_{v}} \Rightarrow \mu \lambda \leq \frac{4 B_{m}\left(B_{s}+K_{v}\right)}{B_{s}} \\
\mu \lambda \leq \frac{B_{m}\left(B_{s}+K_{v}\right)^{2}-2\left(K_{p}+K_{e}\right) M_{s} B_{m}}{M_{s} K_{v} K_{e}} \\
\mu \lambda \leq \frac{B_{m}\left(B_{s}+K_{v}\right)}{M_{s} K_{e} K_{v}^{2}}\left(\left(B_{s}+K_{v}\right) K_{v}-2 M_{s} K_{p}+2 \sqrt{M_{s}^{2} K_{p}^{2}+M_{s} K_{v}^{2} K_{e}-K_{p} K_{v} B_{s} M_{s}}\right) \\
\mu \lambda \geq \frac{B_{m}\left(B_{s}+K_{v}\right)}{M_{s} K_{e} K_{v}^{2}}\left(\left(B_{s}+K_{v}\right) K_{v}-2 M_{s} K_{p}-2 \sqrt{M_{s}^{2} K_{p}^{2}+M_{s} K_{v}^{2} K_{e}-K_{p} K_{v} B_{s} M_{s}}\right)
\end{gathered}
$$

\section{REFERENCES}

[1] D. Lawrence, "Stability and transparency in bilateral teleoperation," IEEE transactions on robotics and automation, vol. 9, no. 5, pp. 624637, October 1993.

[2] K. Fite, L. Shao, and M. Goldfarb, "Loop shaping for transparency and stability robustness in bilateral telemanipulation," IEEE Transactions on Robotics and Automation, vol. 20, no. 3, pp. 620-624, June 2004.

[3] T. Brooks, "Telerobotic response requirements," in Proceedings of the IEEE International Conference on Systems, Man and Cybernetics, Los Angeles, USA, November 1990, pp. 113-120.

[4] K. Hashtrudi-Zaad and S. Salcudean, "Analysis of control architectures for teleoperation systems with impedance/admittance master and slave manipulators," The international Journal of Robotics Research, vol. 20, no. 6, pp. 419-445, June 2001.

[5] Y. Yokokohji and T. Yoshikawa, "Bilateral control of master-slave manipulators for ideal kinesthetic coupling," IEEE transactions on robotics and automation, vol. 10, no. 5, pp. 605-620, October 1994.

[6] B. Hannaford, "A design framework for teleoperators with kinesthetic feedback," IEEE Transactions on Robotics and Automation, vol. 5, no. 4, pp. 426-434, August 1989.

[7] M. Cavusoglu, A. Sherman, and F. Tendick, "Bilateral controller design for telemanipulation in soft environments," IEEE Transactions on Robotics and Automation, vol. 18, no. 4, pp. 641-647, August 2002.

[8] G. De Gersem, H. Van Brussel, and F. Tendick, "Reliable and enhanced stiffness perception in soft-tissue telemanipulation," The international Journal of Robotics Research, vol. 24, no. 10, pp. 805822, October 2005.
[9] M. Tavakoli, A. Aziminejad, R. Patel, and M. Moallem, "Enhanced transparency in haptics-based master-slave systems," in Proceedings of the 2007 American Control Conference, New York City, USA, July 2007, pp. 1455-1460.

[10] I. Aliaga, A. Rubio, and E. Sanchez, "Experimental quantitative comparison of different control architectures for master-slave teleoperation," IEEE Transactions on Control Systems Technology, vol. 12, no. 1, pp. 2-11, January 2004.

[11] B. Hannaford and R. Anderson, "Experimental and simulation studies of hard contact in force reflecting teleoperation," in Proceedings of the IEEE International Conference on Robotics and Automation, Phil., PA, 1988.

[12] R. Daniel and P. McAree, "Fundamental limits of performance for force reflecting teleoperation," The International Journal of Robotics Research, vol. 17, no. 8, pp. 811-830, August 1998.

[13] N. Hogan, "Controllling impedance at the man/machine interface," in Proceedings of the IEEE International Conference on Robotics and Automation, Scottsdale, AZ, 1989, pp. 1626-1631.

[14] J. Colgate and N. Hogan, "Robust control of dynamically interacting systems," International Journal on Control, pp. 65-88, 1988.

[15] J. E. Colgate, "Robust impedance shaping telemanipulation," IEEE Transactions on robotics and automation, vol. 9, no. 4, pp. 374-384, August 1993.

[16] F. Llewellyn, "Some fundamental properties of transmission systems," in Proc. IRE, vol. 40, 1952, pp. 271-283.

[17] R. Adams and B. Hannaford, "Stable haptic interaction with virtual environments," IEEE transactions on robotics and automation, vol. 15, no. 3, pp. 465-474, June 1999.

[18] A. Okamura, "Methods for haptic feedback in teleoperated robotassisted surgery," Industrial Robot: An international Journal, vol. 31, no. 6, pp. 499-508, 2004.

[19] S. Misra and A. Okamura, "Environment parameter estimation during bilateral telemanipulation," in Proceedings of Symposium on Haptic Interfaces for Virtual Environment and Teleoperator Systems, Virginia, USA, March 2006, pp. 301-307.

[20] B. Willaert, E. Vander Poorten, D. Reynaerts, and H. Van Brussel, "Reliable stiffness reflection for telesurgery," in ICRA 2008 Workshop: New Vistas and Challenges in Telerobotics, Pasadena, California, USA, 2008.

[21] H. I. Son and D. Y. Lee, "Enhancement of kinesthetic perception for microsurgical teleoperation using impedance-shaping," in Proceedings of the 30th Annual Int. IEEE EMBS Conference, Vancouver, B.C., Canada, August 2008, pp. 1939-1942.

[22] C.R.Doarn, K.Hufford, T. L. J. Rosen, and B.Hannaford, "Telesurgery and robotics: A roundtable discussion." Telemed J E Health, vol. 13, no. 4, pp. 369-380., 2007.

[23] M. J. Lum, J. Rosen, T. S. Lendvay, M. N. Sinanan, and B. Hannaford, "Effect of time delay on telesurgical performance," in Proceedings of the International Conference on Robotics and Automation, Kobe, Japan, May 2009.

[24] E. Vander Poorten, "Improving haptic fidelity for intuitive manipulation in scaled teleoperation and virtual environment," Ph.D. dissertation, University of Kyoto, 2007.

[25] H. Cho and J. Park, "Impedance control with variable damping for bilateral teleoperation under time delay," JSME International Journal Series $C$, vol. 48, no. 4, pp. 695-703, 2005.

[26] A. Haddadi and K. Hashtrudi-Zaad, "A new robust stability analysis and design tool for bilateral teleoperation control systems," in Proceedings of the IEEE International Conference on Robotics and Automation, Pasadena, CA, USA, May 2008, pp. 663-670.

[27] K. Fite, J. Speich, and M. Goldfarb, "Transparency and stability robustness in two-channel bilateral telemanipulation," Journal of Dynamic Systems, Measurement, and Control, vol. 123, pp. 400-407, 2001.

[28] Y. Bu, R. Daniel, and P. McAree, "Stability analysis of force reflecting telerobotic systems," in Proceedings of IEEE International Conference on Intelligent Robots and Systems, vol. 3, Osaka, Japan, November 1996, pp. 1374-1379.

[29] J. E. Colgate, "The control of dynamically interacting systems," Ph.D. dissertation, Massachussetts Institute of Technology, August 1988.

[30] W. S. Newman, "Stability and performance limits of interactions controllers," Journal of dynamic systems, measurement, and control, vol. 114, no. 4, pp. 563-570, 1992.

[31] S. Haykin, "Active network theory," Addison-Wesley, 1970. 